\title{
Determination of UV-visible-NIR absorption coefficient of graphite bulk using direct and indirect methods
}

\author{
T. Smausz ${ }^{1,2}$, B. Kondász ${ }^{2}$, T. Gera ${ }^{2}$, T. Ajtai ${ }^{1}$, N. Utry ${ }^{2}$, M. Pintér ${ }^{2}$, G. Kiss-Albert ${ }^{2}$, J.

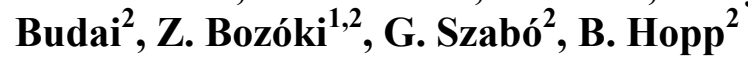 \\ ${ }^{1}$ MTA-SZTE Research Group on Photoacoustic Spectroscopy, University of Szeged, H-6720 \\ Szeged, Dóm tér 9, Hungary \\ ${ }^{2}$ Department of Optics and Quantum Electronics, University of Szeged, H-6720 Szeged, Dóm \\ tér 9, Hungary
}

Corresponding author: T. Smausz tomi@physx.u-szeged.hu

\begin{abstract}
Absorption coefficient of graphite bulk pressed from 1-5 micrometers size crystalline grains was measured in UV-Vis-NIR range with three different methods: i) determination of pulsed laser ablation rate as the function of laser fluence for different wavelengths ' $(248 \mathrm{~nm}, 337 \mathrm{~nm}$, $532 \mathrm{~nm}$ and $1064 \mathrm{~nm}$, respectively); ii) production of aerosol particles by UV laser ablation of the bulk graphite in inert atmosphere and determination of the mass-specific absorption coefficient with a four-wavelength $(266 \mathrm{~nm}, 355 \mathrm{~nm}, 532 \mathrm{~nm}$ and $1064 \mathrm{~nm}$, respectively) photoacoustic spectrometer and iii) spectroscopic ellipsometry in 250-1000 nm range. Taking into account the wide range of the absorption coefficients available in for the different carbon structures, an overall relatively good agreement was observed for the three methods. The ellipsometric results fit well with the ablation rate measurement, the data obtained with photoacoustic method are also similar in the UV and NIR region, however the values were somewhat higher in visible and near-UV range. Taking into account the limitations of the methods, they can be promising candidates for the determination of absorption coefficient when the samples are strongly scattering and there is no possibility to perform transitivity measurements.
\end{abstract}

Keywords: graphite, absorption coefficient, laser ablation, photoacoustic spectroscopy, ellipsometry 


\section{Introduction}

The determination of absorption coefficient for powder pressed or highly absorptive materials with porous structure is not always a straightforward task. When the thickness of the sample does not allow transmission measurement, the measurement of diffuse reflectance can give information on the absorptive properties. The interpretation of the reflectance data require the application of theoretical models (Kubelka-Munk, statistical theories, etc.) combined with assumptions on the target properties (grain size, refractive index, etc.), however the results obtained have a relatively high uncertainty especially if the reflectance is very high (above $\sim 80 \%$ ) or very low (under $10-20 \%$ ) $[1,2]$. It is well known that carbon samples have low reflectivity/high absorption, therefore, besides the reflectometry measurements, the absorption coefficient was also measured on ultrathin sections or deposited thin films [3-7]. Nevertheless, the studies showed that the optical properties of carbon layers are strongly influenced by the method the samples were prepared.

There are indirect methods which are also able to give information on the absorptive properties of materials. One of them is the laser ablation when a focused pulsed laser beam is used to explosively remove the irradiated volume from the target. The ablation rate (i.e. thickness removed per pulse) is proportional to the penetration depth of the light at the given wavelength. The penetration depth is the reciprocal of the effective absorption coefficient, which corresponds to the linear absorption coefficient if some conditions stand: the target material undergoes photochemical decomposition, negligible incubation effect and low heat diffusion from the irradiated volume [8]. The wavelength dependence of the ablation process of graphite was studied earlier for a wide fluence range [9] without focusing onto the neighborhood of the threshold fluence, which would important if using the data for absorption coefficient determination.

Utilizing the fact that bulk absorptive properties can also be deduced from its particulate phase, the other useful indirect methodology for determination of that is the particle phase photoacoustic spectroscopy [10]. The photoacoustic spectroscopy measure the absorption coefficient of aerosol assembly dispersed in a gas ambient through free-floating (in-situ) sampling, therefore it is not suffered any measurement artefact associated with the transmission measurement of filter accumulated aerosol. Nevertheless, the PA techniques have other two great benefits against the alternatives. One is that it is completely insensitive for light scattering [11]. The other is that it is a so called zero background techniques (no excitation-no signal), which makes it capable to determine light absorption by aerosol with high sensitivity even in its low mass concentration [12,13]. Because of these there is a widely accepted consensus in that the PA technique is most suitable methodology for aerosol absorption measurement today. In our earlier works the laser ablation was used to produce aerosols from coal targets where optical absorption coefficient of the aerosol particles was successfully monitored with a four-wavelength photoacoustic system $[14,15]$. The motivation of such studies was that the anthropogenic carbon-containing particles are constituents of atmospheric aerosols with healthcare risk and significant contribution to their climate forcing.

Spectroscopic ellipsometry is a flexible tool in the determination of optical properties of various material structures. It is based on the measurement of the change in polarization state of light when reflected from the sample. Although the optimal samples show specular reflection, with the use of proper correction methods developed recently, samples with significant diffuse scattering can also be measured [16,17].

The aim of our study was to determine the optical absorption coefficient of high purity amorphous graphite with two indirect methods (ablation rate measurement of bulk and photoacoustic measurement on the so produces aerosols) and the spectroscopic ellipsometry and to compare of the results with regard to the applicability and limitations of the methods. 


\section{Experiments}

\subsection{Absorption coefficient determined on the basis of ablation rate equation}

The ablation rate of graphite (Goodfellow, 99.95\% purity, pressed from 1-5 $\mu \mathrm{m}$ size crystalline grains) was measured for four different wavelengths. Before the irradiation the surface of disk shaped graphite targets was polished, the roughness was $0.5 \mu \mathrm{m}$. The $248 \mathrm{~nm}$ irradiation was performed with $\mathrm{KrF}$ excimer laser ( $\mathrm{FWHM}=18 \mathrm{~ns}$ ). An aperture was used to select a homogeneous part of the laser beam and the aperture was projected onto the surface of the target as to obtain a nearly tophat fluence distribution. The size of the irradiated spot was $450 \mu \mathrm{m}$ and the applied number of pulses was 2000 . The pulse energy was measured shot by shot, averaged and divided by the spot size to obtain the actual fluence. Irradiation at 337 nanometer wavelength was performed with a medium pressure $\mathrm{N}_{2}$ laser having $1 \mathrm{~ns}$ pulse length, while beam of a second harmonic and fundamental Nd:YAG laser (FWHM=8 ns) was used for $532 \mathrm{~nm}$ and $1064 \mathrm{~nm}$ irradiation. For the two latter lasers we could not obtain homogeneous irradiation spot, therefore their average fluence values can be compared only within the individual experimental series. The pulse-to pulse fluctuation of the energy was within $15 \%$, and the energy measurement accuracy was $\sim 5 \%$ for all the lasers used. The etched depths were measured a Dektak8 surface profiler, and the calculated etch rates (depth ablated per one pulse) were calculated for each parameter set.

\subsection{Photoacoustic measurement of absorption coefficient}

A 4-wavelength photoacoustic (PA) device (MuWaPaS: Multi-Wavelength Photoacoustic Spectrometer) was applied for determination of the absorption coefficient of graphite aerosols generated via UV laser ablation, as follows. The graphite target was placed in a chamber and the beam of the $\mathrm{KrF}$ excimer laser was focused onto its surface (Fig. 1). The size of the irradiated spot, the average fluence (nearly Gaussian spatial distribution) and the repetition rate were $2 \mathrm{~mm}^{2}, 2.5 \mathrm{~J} / \mathrm{cm}^{2}$ and $1 \mathrm{~Hz}$, respectively. The ablation generated graphite particles were collected by high purity nitrogen flowed through the chamber and transported into the analyzing cells of our PA measuring system. Nitrogen was selected as carrier gas to avoid gas-phase cross sensitivity to ozone at $266 \mathrm{~nm}$ and also to make the particle evolvement more efficient [18]. The four individual photoacoustic cells containing the ablatum-nitrogen mixture were irradiated with one of the four measuring wavelengths $(266,355,532$ and 1064 $\mathrm{nm}$, respectively) simultaneously, and the PA response was measured in each cell. The mass specific absorption cross section $\left(\sigma_{p a}\right)$ of the graphite particles can be determined according to the following equation of the PA signal strength $(S)$ :

$S=\sigma_{p a} \cdot \gamma_{A} \cdot C_{c e l l} \cdot P_{\text {laser }}+B C_{p}$,

where $\mathrm{C}_{\text {cell }}$ is the PA setup constant which represents the PA response generated by unit laser power absorbed by unit optical absorption of the studied material $\left(\mathrm{nV} /\left(\mathrm{m}^{-1} \times \mathrm{mW}\right)\right), P_{\text {laser }}$ is the power of the probe lasers in $\mathrm{mW}$ and $\gamma_{A}$ is the mass concentration of the aerosol loaded gas sample in $\mathrm{g} / \mathrm{m}^{3}$, and $B C_{p}$ is the background signal. The $C_{\text {cell }}$ is determined through independent gas phase calibration procedure, while the $P_{\text {laser }}$ is measured continuously during the measurement. Therefore, through the parallel measurement of signal $S$ the $\sigma_{p a} \gamma_{A}$ product, In order to increase the accuracy of the measurement the background (aerosol-free) signal was measured from time to time using a high-efficiently particle (Balston DFU) filter. The measurement system and protocol, as well as the calibration procedure are described in detail elsewhere [14,15]. To deduce the $\sigma_{p a}$ the $\gamma_{A}$ mass concentration of the aerosol was measured simultaneously on the same aerosol assembly by a tapered element oscillating microbalance (TEOM, Rupprecht and Patashnick, Model 1400a). 


\subsection{Ellipsometric measurements}

Measurements were performed on polished and unpolished parts of the sample at different points using a Semilab SE1000 rotating compensator ellipsometer in 250-1000 nm wavelength range. Ellipsometric data were collected at two different angles of incidence $\left(70^{\circ}\right.$ and $75^{\circ}$ ) applying focusing optics. The measured data could be directly transformed to complex refractive index values, however to reduce noise level an oscillator based fitting was applied. Details of the fittings are described in the Results section.

\section{Results}

The ablation rates were calculated for all the four wavelengths and plotted as the function of laser fluence (Fig. 2). The most detailed study performed at $248 \mathrm{~nm}$ wavelength showed that there is a $\sim 15 \%$ deviation of the ablation rate values obtained for the same fluence value. It can be seen that the ablation rate starts to increase from 0 at around $1000 \mathrm{~mJ} / \mathrm{cm}^{2}$, however this low fluence range ablation process differs from the high fluence range and the depth in this regime is not strictly related to the absorption coefficient. Therefore the well-known formula for ablation rate was fitted to the measured data points for fluences above the high fluence range ablation threshold $\left(\sim 2500 \mathrm{~mJ} / \mathrm{cm}^{2}\right)$ :

$d=\frac{1}{\alpha_{a b l}} \ln \frac{F}{F_{t h}}$, where the $\alpha_{a b l}$ is the effective absorption coefficient and the $F_{t h}$ threshold fluence were considered as fitting parameters. For $248 \mathrm{~nm}$ wavelength the obtained value for $\alpha_{a b l}$ and $F_{t h}$ were $15.27 \pm 0.92 \mu \mathrm{m}^{-1}$ and $1914 \pm 110 \mathrm{~mJ} / \mathrm{cm}^{2}$. For $337 \mathrm{~nm}, 532 \mathrm{~nm}$ and $1064 \mathrm{~nm}$ wavelengths the so calculated absorption coefficient values were $9.91 \pm 0.98 \mu \mathrm{m}^{-1}, 4.67 \pm 0.47$ $\mu \mathrm{m}^{-1}$ and $4.56 \pm 0.39 \mu \mathrm{m}^{-1}$, respectively (Table 1 ). The fluence values for these three wavelengths were in arbitrary units only.

The $\sigma_{p a}$ mass-specific optical absorption coefficient of laser produced graphite aerosol was calculated on the basis of PA signal strength of the four applied wavelengths and the measured mass concentration. The $\alpha$ linear absorption coefficient can be calculated as the product of mass-specific absorption coefficient and the $\rho$ density of the material: $\alpha=\sigma \cdot \rho$. We measured the density of our bulk graphite sample and is was found to be $1.72 \mathrm{~g} / \mathrm{cm}^{3}$, much less than the theoretical the value of $2.26 \mathrm{~g} / \mathrm{cm}^{3}$ indicating that the sample contains considerable amount of voids and/or is formed by less compact atomic arrangements. The so derived $\alpha_{p a}$ absorption coefficient values are also summarized in Table 1. By fitting an exponential function to the PA derived data, the $\alpha_{p a}$ values for the ablating laser wavelengths could be estimated by interpolation/extrapolation.

The ellipsometric data measured on the polished and unpolished surfaces differed not only in their actual values, but also in their noise level. Due to the enhanced scattering of the unpolished surfaces the intensity of the reflected light was decreased resulting in higher noise levels. Therefore, before fitting the complex refractive index measured on the unpolished surfaces first the data obtained on the polished top surface were analyzed. For this purpose a one-phase model was used, where the dispersion of the carbon material was described using Gaussian-, Drude-, and Sellmeier oscillators [19,20]. When proceeding to the data recorded on the unpolished surfaces, the broadening and position parameters of these oscillators were kept fixed and only the amplitude parameters were fitted. The deduced complex refractive indices are presented in Fig. 3. The uncertainty of the optical functions indicated with gray regions around the curves was determined as the scattering of the data belonging to different sample positions. While in the case of the polished surface nearly the same extinction coefficients were measured for the three different locations, the data of the unpolished surfaces exhibited more differences. The tendencies of both curves are similar to that of the 
basic carbon forms (graphite and glassy carbon) available in the literature, however the values are smaller, which indicate a porous material structure. The increase in the $n$ and $k$ values and thus the decrease in the porosity by polishing the surface is even more pronounced. This suggests that although the nanoscale porosity of the target forming grains persists, the polishing increases the local compactness of the sample by filling the voids existing between the grains, resulting in higher $\mathrm{n}$ and $\mathrm{k}$ values. Since from the point of view of the ablation and photoacoustic measurements the bulk or effective properties of the carbon target play important role, in the followings only the results of the unpolished surfaces will be discussed. From the $k$ values the $\alpha_{\text {ellips }}$ absorption coefficient was calculated using the $\alpha=4 \pi k / \lambda$ formula and the values for the wavelengths used for the other measurements are also included in Table 1.

Fig. 4 shows the wavelength dependence of the absorption coefficients measured with the three different methods. Taking into account that there can be orders of magnitude spread in in the absorption coefficients of the different carbon structures, it is clearly visible that there is relatively good agreement between the data obtained on our sample by direct (ellipsometry) and indirect (photoacoustic and ablation) methods. At this point we have to note, that the wavelength dependence of the optical absorption of the graphitic or turbostratic, pure elemental carbon structure is expected to have a decreasing power function $\left(\alpha \sim \lambda^{-p}\right.$, where $p$ is called speed of absorption) in the whole visible-near IR region and to deviate from this (steeper slope, increase of speed) in the UV range. The PA spectra fulfill this criteria in the visible-near IR region, but we did not observe the expected quick change in the, while the ablation and the ellipsometric measurement results are indicating much moderate wavelength dependency in the visible-near IR, and continuously increased absorption speed towards the shorter wavelengths.

\section{Discussions}

A comparison of the three methods is possible only if similar graphitic structures are measured and the measured properties are identical. According to the information given by Goodfellow the grains of the graphite rod are crystals with a typical hexagonal atomic structure. As our earlier studies showed that the aerosol particles produced with present experimental conditions are fractal aggregates showing unimodal distribution with a count median diameter of $150 \mathrm{~nm}$ and $160 \mathrm{~nm}$ FWHM, while high resolution transmission electron microscopic and Raman spectroscopic investigations showed that the particles mainly consist of graphitic atomic arrangement structures [18]. Besides, in the literature, for high purity graphite aerosol particles produced in flame or electric discharge the value of $1.8 \mathrm{~g} / \mathrm{cm}^{3}$ for the inherent particle density is widely accepted, which is also very close to that of our graphite sample. Fig. 5 shows an electron microscopic image of a freshly broken surface of the graphite rod, one can see that the target is formed by relatively compact irregularly shaped grains. Larger voids with sizes above the wavelengths used in our measurements are not dominantly present. From the point of view of the measurements it is important that the presence of fine porosity (with characteristic sizes below the wavelength) makes the sample to behave as bulk with an effective complex refractive index.

The applied methods used completely different approach for the absorption coefficient determination. The ablative and photoacoustic methods are based on light penetration and the measurement of effects related to the absorbed light energy. In the ablation rate function the $\mathrm{F} / \mathrm{F}_{\text {th }}$ ratio is independent from the reflectivity of the sample, the other factor influencing it is grade of intensity attenuation $\left(\alpha_{a b l}\right)$ as the light penetrates the sample. The total ablation depth was much larger, while the ablation rate was one or two orders of magnitudes smaller than the grain size, therefore the grainy structure should not influence the ablation process and (e.g. by grain expulsion) $\alpha_{a b l}$ is also an averaged value of the porous graphite target. 
In case of photoacoustic measurement the modulated light intensity is absorbed in the aerosol particles and transformed into thermal energy creating a periodical pressure wave (acoustic signal) in the measuring cell. The size of the produced aerosol particles is mainly in the Rayleigh regime, besides, such carbon particles behave as volume absorbers [21] (absorption is not localized on the surface of particles) therefore the obtained $\sigma_{\mathrm{pa}}$ can be considered material specific for the aerosol constituents. Thus, the derived $\alpha_{\mathrm{pa}}$ corresponds to the bulk graphite target.

Since the graphite rod was produced by high pressure compaction of the grains, it is plausible that at the surface the ellipsometer detected a strongly scattering rough surface created by the individual 1-5 micrometer sized grains, however when eliminating the surface scattered light, the fine porosity structure allows the target to behave as a bulk exhibiting an effective linear absorption coefficient.

Taking into account the large spread of the absorption data available in the literature for the various types carbon materials obtained with different methods, we can affirm that our results obtained for the three different methods are in relatively good agreement. The measurement error was the highest for ellipsometric method, however its average values fit well with those obtained with ablation rate measurement. Similar results were obtained with photoacoustic method in the UV and NIR region, however the values were somewhat higher in visible and near-UV range. Although all the measurements have they own uncertainty originating from different influencing factors, this tendentious discrepancy can be caused either from the presence of larger fissures (up to about $1 \mu \mathrm{m}$ ) in the bulk or somewhat altered atomic level structure of the aerosol particles as compared to the starting material.

\section{Conclusions}

In the present work we successfully applied three different methods for the determination of absorption coefficient of highly absorptive graphite bulk. Taking into account the limitations of the methods, they can be applied for the absorptivity measurement when the samples are strongly scattering and there is no possibility to perform transmission measurements. The ablation rate method can be applied when the incubation effect is not present and thermal conductivity does not play important role in the material removal. Crumbling of the ablated sample would also result in apparently higher ablation rate. The aerosol production with laser irradiation has also its limitations, since there can be mismatch between the atomic structure and absorptive properties of the aerosol and target material. However, in case of elemental carbon many earlier studies have confirmed experimentally that the ablated aerosol nanostructure is inherited by the bulk sample. We believe that the PA method could be applied for other materials, too, if proper fragmentation and dispersing methods are applied. Our measurements also showed that the ellipsometric method initially developed for specular reflection, with the use of proper sampling and analysis methods can be used in case of highly absorptive and strongly scattering bulks.

\section{Acknowledgements}

This work was supported by the GINOP-2.3.2-15-2016-00036 ("Development and application of multimodal optical nanoscopy methods in life and material sciences") project.

\section{References}

1. H.G. Hecht, J. Res. NBS A Phys. Ch. 80A, 567 (1976)

2. P.D. Johnson, J. Opt. Soc. Am. 42, 978 (1952)

3. J.T. Mc-Cartney, S. Ergun, US Bur. Mines Bull. 641, 1 (1967) 
4. N. Laidani, R. Bartali, G. Gottardi, M. Anderle, Pierre Cheyssac, J. Phys.-Condens. Mat. (2008) doi:10.1088/0953-8984/20/01/015216

5. T. Kaplas, L. Karvonen, J. Rönn, M. R. Saleem, S. Kujala, S. Honkanen, Y. Svirko, Opt. Mater. Express 2, 1822 (2012)

6. S. Logothetidis, Diam. Relat. Mater. 12, 141 (2003)

7. P.K. Chu, L. Li, Mater. Chem. Phys. 96, 253 (2006)

8. T. Lippert, Adv. Polym. Sci. 168, 51 (2004)

9. J. Hoffman, J. Chrzanowska, S. Kucharski, T. Moscicki, I.N. Mihailescu, C. Ristoscu, Z. Szymanski, Appl. Phys. A 117, 395 (2014)

10. H. Moosmüller, R.K. Chakrabartry, K.M. Ehlers, W.P. Arnott, Atmos. Chem. Phys. 11, 1217 (2011)

11. W.P. Arnott, K. Hamasha, H. Moosmüller, P.J. Sheridan, J.A. Ogren, Aerosol Sci. Tech. 39, 17 (2005)

12. B.A. Flowers, M.K. Dubey, C. Mazzoleni, E.A. Stone, J.J. Schauer, S.-W. Kim, Atmos. Chem. Phys. 10, 10387 (2010)

13. N. Utry, T. Ajtai, Á. Filep, M. Pintér, Z. Török, Z. Bozóki, G. Szabó, Atmos. Environ. 91, 525 (2014)

14. T. Ajtai, Á. Filep, M. Schnaiter, C. Linke, M. Vragel, Z. Bozóki, G. Szabó, T. Leisner, J. Aerosol Sci. 41, 1020 (2010).

15. T. Ajtai,'Á. Filep, 'G. Kecskeméti, B. Hopp, ZZ. Bozóki,·G. Szabó, Appl. Phys. A 103, $1165(2011)$

16. Z. Pápa, J. Budai, I. Hanyecz, J. Csontos, Z. Toth, Thin Solid Films 571, 562 (2014)

17. M. Ben-David, L. Engel, Y. Shacham-Diamand, Microelectron. Eng. 171, 37 (2017)

18. T. Ajtai, N. Utry, M. Pintér, G. Kiss-Albert, R. Puskás, Cs. Tápai, G. Kecskeméti, T. Smausz, B. Hopp, Z. Bozóki, Z. Kónya, G. Szabó, Atmos. Meas. Tech. 8, 1207 (2015)

19. H. Fujiwara, Spectroscopic Ellipsometry, Principles and Applications, (John Wiley Sons Inc., New York, 2007), pp. 158-176

20. H.G. Tompkins, J.N. Hilfiker, Spectroscopic Ellipsometry, (Momentum Press, LLC, New York, 2016)

21. H. Moosmüller, R.K. Chakrabarty, W.P. Arnott, J. Quant. Spectrosc. Ra. 110, 844 (2009) 


\section{Tables}

Table 1 Summary of the absorption coefficients measured with different methods.

\begin{tabular}{|l|l|l|l|l|l|l|}
\hline Wavelength [nm] & 248 & 266 & 337 & 355 & 532 & 1064 \\
\hline $\begin{array}{l}\text { Photoacoustic } \\
\alpha_{\mathrm{pa}}\left[\mu \mathrm{m}^{-1}\right]\end{array}$ & $16.77^{*}$ & 15.9 & $12.4^{*}$ & 11.76 & 7.7 & 3.71 \\
\hline $\begin{array}{l}\text { Ablation } \\
\alpha_{\mathrm{abl}}\left[\mu \mathrm{m}^{-1}\right]\end{array}$ & 15.27 & & 9.91 & & 4.67 & 4.56 \\
\hline $\begin{array}{l}\text { Ellipsometry } \\
\alpha_{\text {ellips }}\left[\mu \mathrm{m}^{-1}\right]\end{array}$ & 18.44 & 16.1 & 8.9 & 8.24 & 5.52 & $3.8^{*}$ \\
\hline
\end{tabular}

inter/extrapolated 


\section{Figure captions}

Fig. 1 Experimental arrangement for aerosol generation and absorption measurements.

Fig. 2 The ablation rate vs. fluence plots of graphite for different laser wavelengths.

Fig. 3 Complex refractive index ( $n$ and $k$ ) measured on polished and original surfaces of the graphite sample.

Fig. 4 Absorption coefficient of graphite measured and calculated with different methods.

Fig. 5 SEM image of a freshly broken graphite surface. 
Fig. 1.

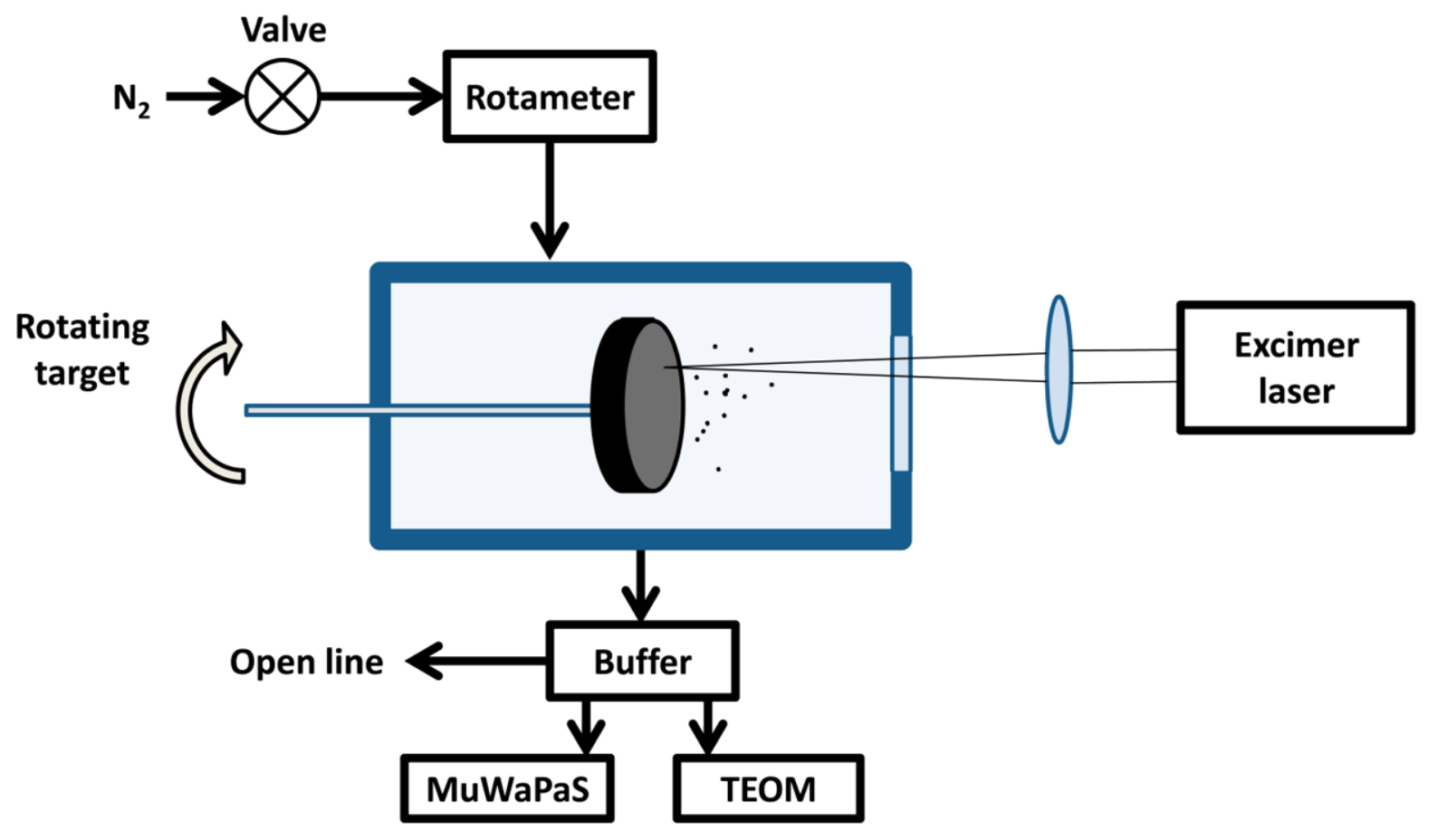


Fig 2.
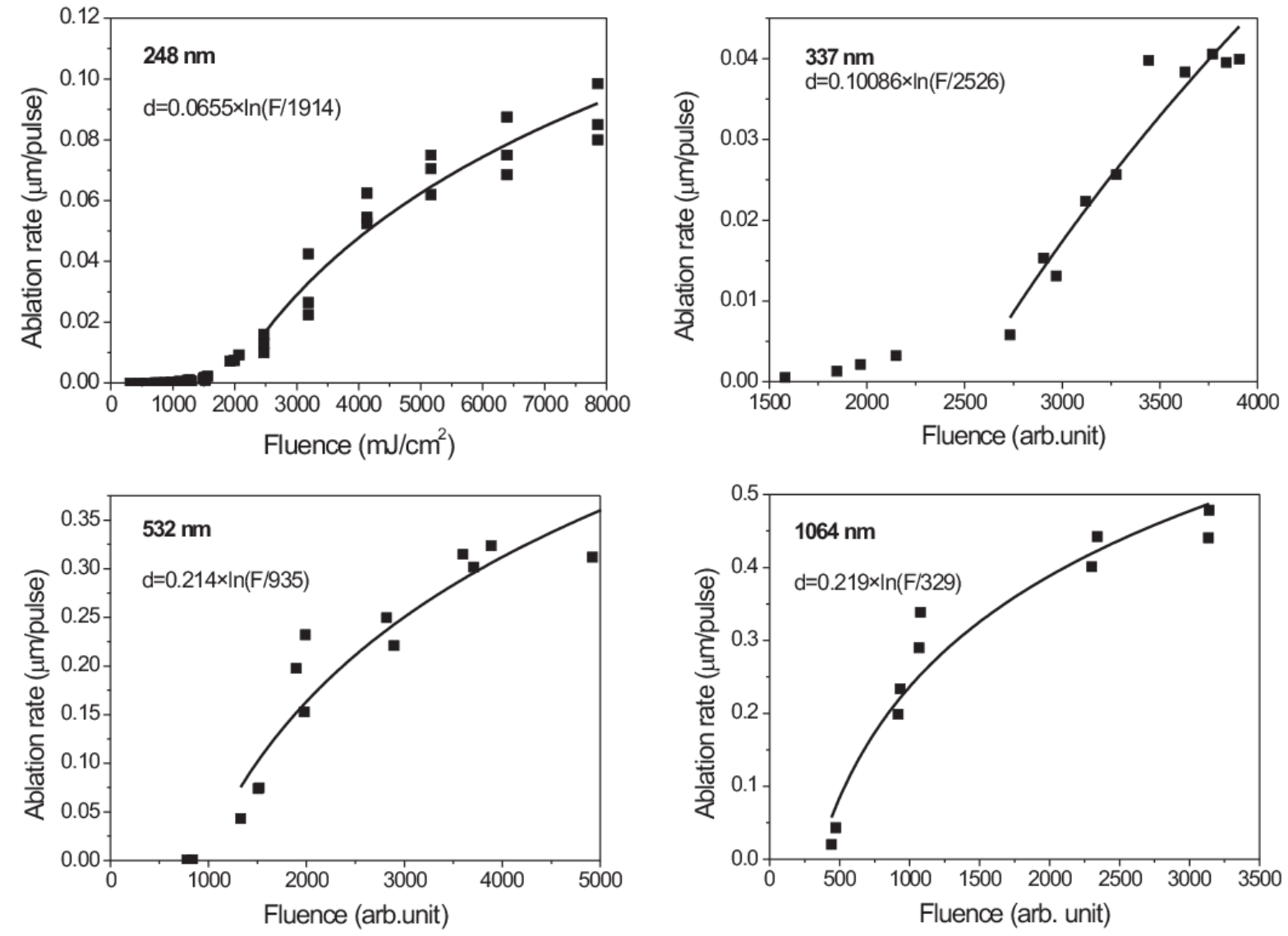
Fig. 3.
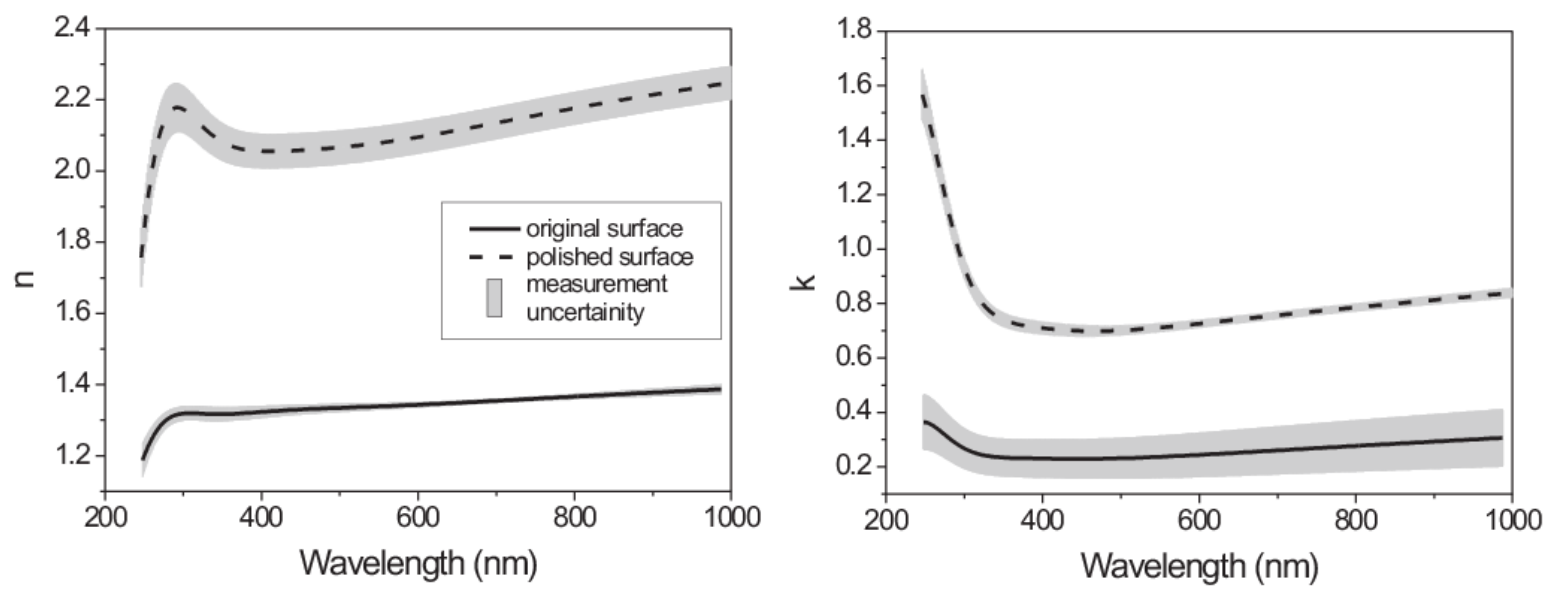
Fig. 4.

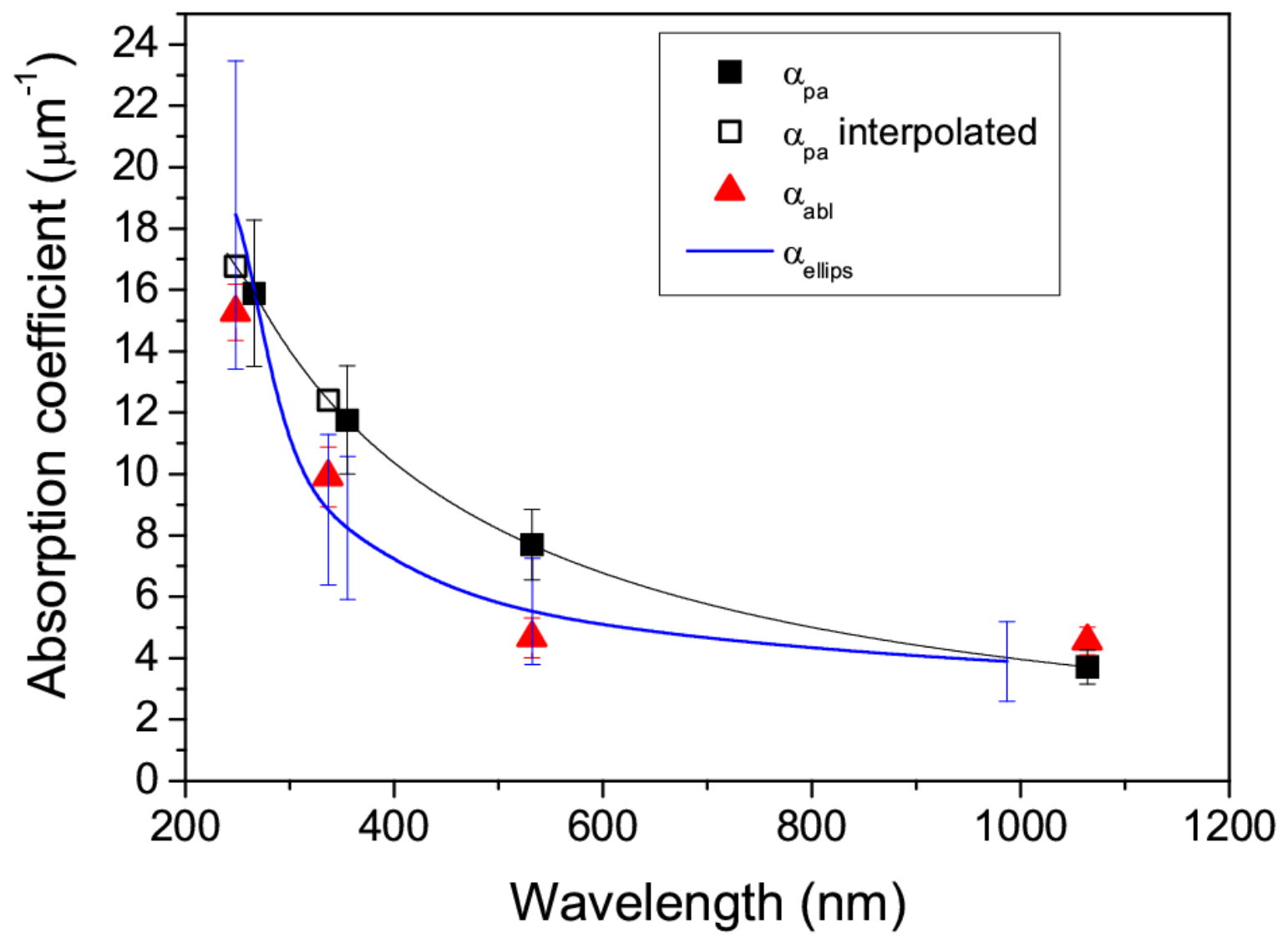


Fig. 5.

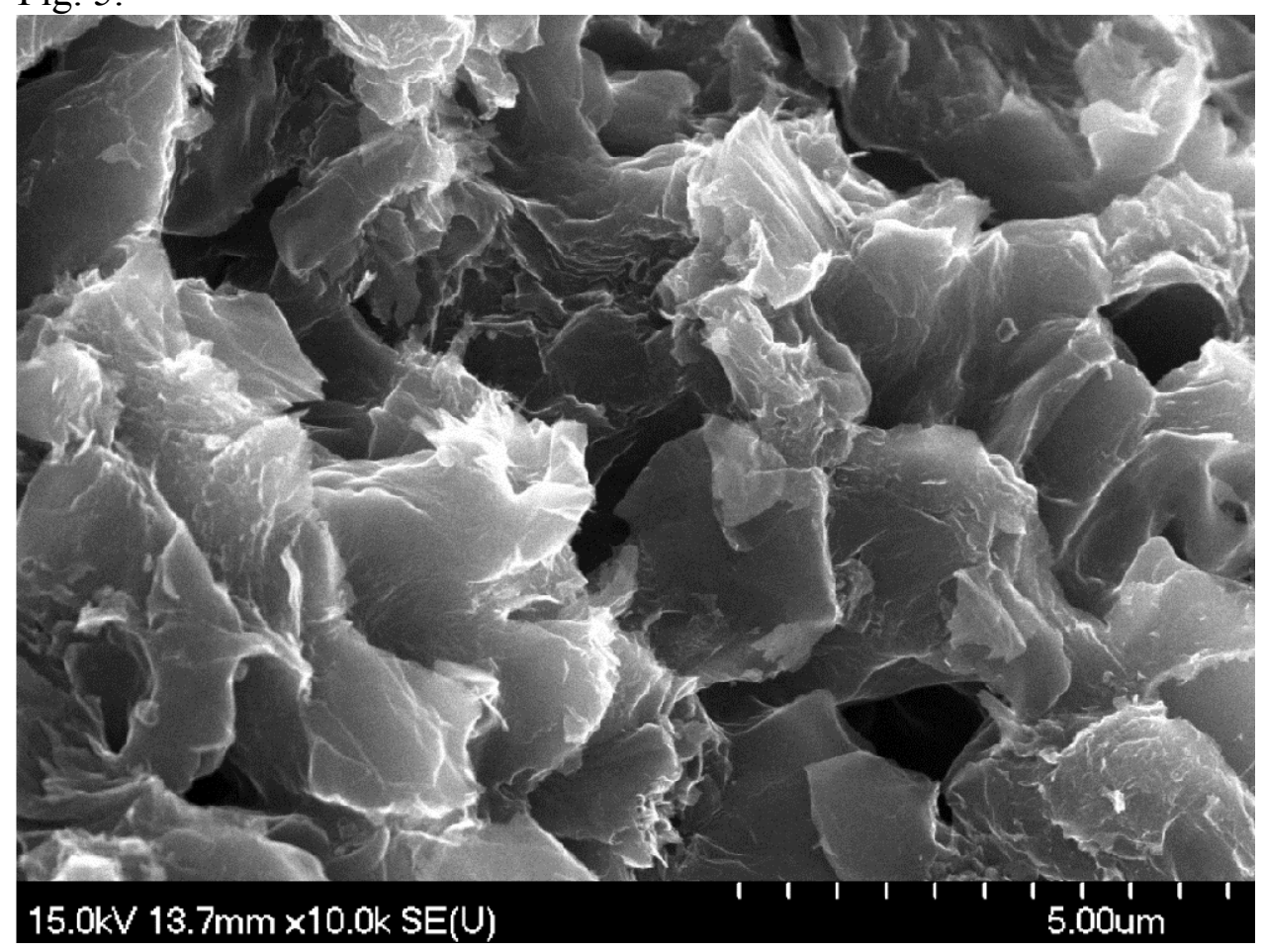

